\title{
The Uterus Sustains Stable Biological Clock during Pregnancy
}

\author{
Shizuko Akiyama, ${ }^{1,2}$ Hidenobu Ohta, ${ }^{1,2,3 *}$ Shimpei Watanabe, ${ }^{1,2}$ Takahiro Moriya, ${ }^{4}$ \\ Aya Hariu, ${ }^{4}$ Norimichi Nakahata, ${ }^{4}$ Hiroshi Chisaka, ${ }^{1,3}$ Tadashi Matsuda, ${ }^{1,2}$ \\ Yoshitaka Kimura, ${ }^{5}$ Shigeru Tsuchiya, ${ }^{3}$ Hajime Tei, ${ }^{6}$ Kunihiro Okamura ${ }^{1,3}$ and \\ Nobuo Yaegashi, ${ }^{1,3}$

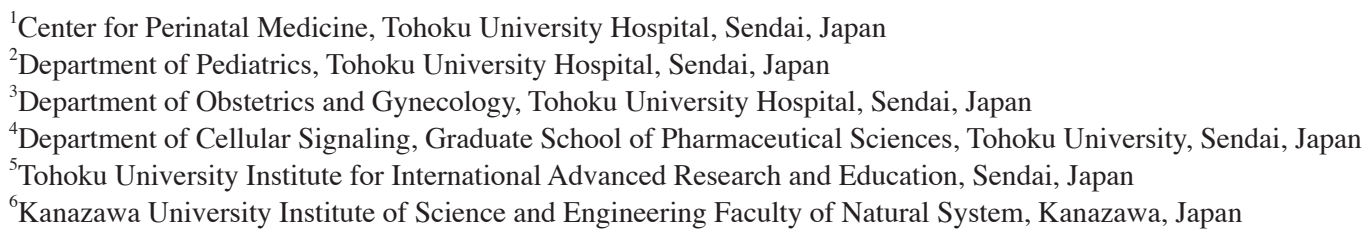

Maternal circadian information has been reported to play an important role in fetal physiology and development. Hormones and nutrition have been mainly investigated as circadian cues from mother to fetus. However, the influences of circadian properties of the pregnant reproductive organs on fetuses have not been fully investigated. To gain an insight on the circadian functions of the reproductive organs, we examined molecular clocks in the pregnant rat uterus and placenta. By using a Period1-/uciferase (Per1-luc) rat, whose tissues express luciferase corresponding to activation of Period1, a "key clock gene", we examined the uterus clock during non-pregnancy, on embryonic day 12 (E12), and on E22 (the end of pregnancy) in a light-dark (LD) cycle and constant darkness (DD). By in situ hybridization we further explored Per1 mRNA rhythms in the placenta on E12 and E22. The uterus in vitro showed clear circadian Per1-luc rhythms both in and out of pregnancy, having peaks at around the time corresponding to dusk in LD. Likewise, in DD, the uterus in vitro had the same Per1-Iuc rhythms. The decidua in LD showed circadian Per1 mRNA rhythms, peaking during night $6 \mathrm{~h}$ after dusk, while the decidua in DD showed the same Per1 mRNA rhythms only on E22. In contrast, the labyrinth showed no circadian Per1 mRNA rhythms in LD or DD during pregnancy. These results suggest that the uterus and decidua, a maternally-originated tissue of the placenta, but not the labyrinth, a fetus-originated tissue of the placenta, can provide the fetus with circadian information.

Keywords: intrauterine environments; circadian; pregnancy; uterus; placenta

Tohoku J. Exp. Med., 2010, 221 (4), 287-298. (C) 2010 Tohoku University Medical Press

Most living organisms exhibit circadian rhythms, regular daily cycles in their behaviors and physiological functions including activity, sleep, metabolism and body temperature, with a cycle length of approximately 24 hours. In mammals, the circadian timing system is organized as a hierarchy of various organ oscillators. Among them the suprachiasmatic nuclei $(\mathrm{SCN})$ of the anterior hypothalamus function as the master pacemaker at the top of the hierarchy, and coordinate clocks in peripheral organs such as the heart, lung, liver, kidney, pancreas and non-pregnant uterus (Hastings et al. 2003; Dolatshad et al. 2006; Maywood et al. 2007). During pregnancy, the fetus itself functions as an organ oscillator in the mother and uses maternal signals to adjust the fetal biological clock to the extrauterine environment (Serón-Ferré et al. 2007; Ohta et al. 2008).

The intrauterine environment is suggested to be a cir- cadian environment in which fetuses are subject to a variety of nonphotic stimuli, including circadian fluctuations in hormonal and nutritional signals from the mother, placental filtering of those signals, and uterine motility (Serón-Ferré et al. 2007; Ohta et al. 2008). In mammals, maternal melatonin has a circadian pattern in its plasma concentration and is thought to convey information on the light-dark cycle to the fetus, thereby synchronizing the developing fetus's biologic clock to the 24-hour cycle (Davis and Mannion 1988; Illnerová et al. 1993; Serón-Ferré et al. 2007). Fetal biological clock has also been reported to be influenced by the maternal feeding schedule during pregnancy (Weaver and Reppert 1986; Ohta et al. 2008). The circadian molecular properties of the pregnant uterus and placenta, however, have not been fully examined in spite of the importance of understanding their circadian effects on fetal physiology.

Received October 13, 2009; revision accepted for publication June 28, 2010. doi:10.1620/tjem.221.287

Correspondence: Hidenobu Ohta, Center for Perinatal Medicine, Tohoku University Hospital, 1-1, Seiryo-machi, Aoba-ku, Sendai,

980-8574, Japan.

e-mail: hideohta@mail.tains.tohoku.ac.jp 
At the molecular level, cellular clocks in the organs are controlled by autoregulatory transcriptional and translational feed back loops of key "clock genes", in which BMAL1 and CLOCK proteins drive expression of the Period (Per) and Cryptochrome (Cry) genes while the PER and CRY proteins in turn suppress the transcription of their own genes (Hastings et al. 2003). To gain an insight on the molecular basis of the circadian stimuli of the intrauterine environment on the fetus, with a transgenic rat model in which the mouse Periodl(Perl) gene promoter has been linked to a luciferase (luc) reporter (Yamazaki et al. 2000), we continuously monitored the expression of Perl, one of the key "clock genes", by recording light emission from uterus and placenta tissues in vitro. By in situ hybridization, we further examined the circadian molecular clocks of the deciduda and labyrinth in the placenta specifically.

\section{Materials and Methods}

\section{Animals and housing}

Homozygous male and female transgenic Per1-luc rats (Japanese Wistar) expressing $6.7 \mathrm{~kb}$ of the mouse Period1(Perl) promoter driving firefly luciferase were mated for this study, causing the Perl-luc rats to become pregnant with homozygous fetuses. Details on the generation of these animals and the methods have been published previously (Hida et al. 2000; Yamazaki et al. 2000). For Per1-luc monitoring from the uterus and placenta tissue, timed-pregnant Perl-luc rats $(n=5)$ were housed individually in cages in a 12:12 h light-dark (LD) cycle (lights on at 08:00h and lights off at 20:00h; 200 lux at cage level during light period) or constant darkness (a dark-dark (DD) cycle) and fed ad lib. Locomotor activity of the rats was recorded with infrared motion sensors using an online system (Actograph System, Rapid Fire Computer, Japan). Timed-pregnant wild-type Wistar rats ( $n=20$ for LD; $n=20$ for DD) were also housed individually in the same manner for in situ hybridization study of the placenta. Animal care and use were reviewed and approved by the Committee for Animal Research of Tohoku University.

Assessment of Perl-luc circadian periods and phases of the uterus, placenta and maternal SCN

Following behavioral assessment to confirm proper circadian locomotor rhythms of animals in a 12:12h LD cycle, non-pregnant Perl-luc female rats were sacrificed for recordings of Perl-luc expressions in the uterus at the diestrous stage $(n=5)$. We sacrificed the non-pregnant rats at Zeitgeber Time (ZT) 11, one hour before lights off (lights on and lights off are defined as ZT0 and ZT12, respectively) and rapidly removed their uterus and suprachismatic nuclei (SCN). In addition, pregnant Per1-luc rats were sacrificed for recordings of Perl-luc expressions in the uterus, the placenta and the SCN either after 12 days of pregnancy (embryonic day 12: E12) $(n=5)$ or 22 days of pregnancy (E22) $(n=5)$ - one day before expected birth (term: 23 days of pregnancy) in the same manner as non-pregnant Per1-luc rats. We sacrificed the pregnant Perl-luc rats at ZT 11 and rapidly removed their uterus, placenta and SCN. Between ZT11 and ZT11.5 the uterine tissue (1-mm thickness) from a uterine horn, the decidua and the labyrinth tissue (1-mm thickness) from the placenta and a pair of SCN tissue sections (coronal sections of 300um thickness, made with a vibroslicer) from the brain of each Perl-luc rat were placed on membrane inserts (Millicell-CM, Millipore, Bedford,
MA) for tissue culture in $1 \mathrm{~mL}$ of medium (Dulbecco's modified Eagle's medium, Sigma, St.Louis, MO) supplemented with $10 \mathrm{mM}$ HEPES (Sigma), 2\% B27, $25 \mathrm{U} / \mathrm{mL}$ penicillin, $25 \mu \mathrm{g} / \mathrm{mL}$ streptomycin, $2.2 \mathrm{mg} / \mathrm{mL} \mathrm{NaHCO} 3,4 \mathrm{mM}$ L-glutamine, and $0.1 \mathrm{mM}$ beetle luciferin (Promega, Madison, WI). Unless noted, medium ingredients were purchased from Invitrogen (Carlsbad, CA). Each culture was sealed in a Petri dish and maintained at $36^{\circ} \mathrm{C}$ in darkness. Bioluminescence was collected in counts per minute for 4.5 days without a medium change using a photomultiplier tube (HC8259MOD, Hamamatsu Corp., Shizuoka, Japan).

To eliminate the effects of the light-dark cycle on the intrauterine organ clocks, another set of non-pregnant $(n=5)$ and pregnant ( $n$ $=5$ for E12; $n=5$ for E22) Per1-luc rats was transferred to constant darkness (DD). On the second day of DD, with the aid of dim red light ( $<0.1$ lux) they were decapitated at circadian time (CT)11 (CT0 and $\mathrm{CT} 12$ are defined as the initiation and termination of locomotor activity in DD, respectively ; CT 0 and 12 approximately correspond to dawn and dusk in the previous LD cycle). Then the tissue culture of each organ and the SCN were prepared in the same way as for the pregnant Per 1-luc rats in the LD cycle.

Photon counts from each culture were detrended with a 2-h running average as described previously (Yamazaki et al. 2000) with modifications using exmax software (gift from Mr. Tuyoshi Yaita and Dr. Shigenobu Shibata, Waseda University, Tokyo, Japan) (Hayasaka et al. 2007; Ohta et al. 2008; Hirao et al. 2009). The period of Per1luc activity (recorded from 12 to $84 \mathrm{~h}$ in vitro) was assessed for each SCN culture and calculated by averaging the period between the first and second peaks and the period between the second and third peaks as previously described (Yamazaki et al. 2000; Ohta et al. 2005). The Perl-luc amplitudes for the uterus and SCN culture were calculated as the difference between the first trough and second peak of the detrended curves of the bioluminescence. Statistical comparisons were performed by one-way ANOVA $(p<0.05)$.

\section{Assessment of Per1 mRNA expression in the placenta}

On E12 and E22, pregnant wild-type Wistar rats were decapitated at each of four circadian phases: ZT0, ZT6, ZT12, and ZT18 ( $n=5$ at each sampling time) in LD. To eliminate the effects of the LD cycle, another set of pregnant wild-type Wistar rats were also transferred to constant darkness (DD) and also decapitated at each of four circadian phases: CT0, CT6, CT12, and CT18 on the second day of DD. During the dark phase, decapitation was performed with the aid of dim red light $(<0.1$ lux). The placenta were quickly removed and frozen in crushed dry ice. The tissue was stored at $-80^{\circ} \mathrm{C}$ until sectioning for in situ hybridization.

In situ hybridization was carried out to determine the level of Perl mRNA in the placenta. The slices (40- $\mu$ m thickness) were taken using a cryostat (HM505E, Microm, Germany). Radiolabelling of the anti-sense and sense cRNA with [RI: a[33P]UTP (New England Nuclear, USA)] and the in situ hybridization was as reported previously (Moriya et al. 2000; Shimomura et al. 2002). Labelled antisense or sense cRNA probes were constructed from restriction enzyme-linearized cDNA templates that were kindly donated by Dr Okamura (Kyoto University). The radioactivity of each slice on BioMax MR film (Kodak) was analysed using a microcomputer interface to an image analysis system (MCID, Imaging Research Inc., Canada) after conversion into optical density by ${ }^{14} \mathrm{C}$-autoradiographic microscales (Pharmacia Biotech, Buckinghamshire, UK). The intensity values of the sections from random coronal sections of the pla- 
centa ( 4 sections per the placenta) were then averaged to calculate the optical density. One-way ANOVA was used to detect the daily oscillation of Periodl mRNA expression in the placenta $(p<0.05)$.

\section{Results}

Circadian rhythms of Perl expression in the rat uterus during pregnancy

Behavioral assessment by infra-red motion sensors confirmed proper circadian locomotor rhythms of pregnant Perl-luc rat mothers, in which rats are more active during the dark period (data not shown) in an LD cycle and also during the subjective dark period (the period corresponding to dark phase in the LD cycle) in constant darkness (data not shown).

In the LD cycle, Perl-luc measurements showed the circadian expressions of the Perl gene in the uterus at both stages of non-pregnancy and pregnancy. Circadian rhythms in Perl-luc activity were apparent in the non-pregnant uterus as previously reported (period: $24.1 \pm 0.8 \mathrm{~h}$, mean \pm S.D., throughout; amplitude: $16,663 \pm 4,262$ photons/min; Fig. 1A, Table 1) (Hastings et al. 2003; Dolatshad et al. 2006; Maywood et al. 2007). Clear Perl-luc circadian rhythms were also observed at the middle of pregnancy, E12 (period: $24.1 \pm 0.5 \mathrm{~h}$; amplitude: $29,686 \pm 14,961$ photons/min; Fig. $1 \mathrm{~B}$, Table 1), and at the end stage of pregnancy, E22, one day before the expected birth (period: $25.1 \pm 0.7 \mathrm{~h}$; amplitude: 19,722 $\pm 10,786$ photons/min; Fig. 1C, Table 1) in LD. In non-pregnant rats, circadian oscillation in the uterus peaked about 3 hours after maternal SCN, indicating that the peripheral tissues such as the uterus responded differently to the light cycle from the maternal SCN. The phase relationship between the uterus and maternal $\mathrm{SCN}$ was also the same in pregnant rats (one-way ANOVA, $p>0.05$, Table 1).

In constant darkness (DD), the same types of results were also obtained: non-pregnant uterus tissues (period: $24.7 \pm 1.1 \mathrm{~h}$; amplitude: 19,013 \pm 11,303 photons/min; Fig. 2A, Table 1), E12 uterus tissues (period: $24.0 \pm 1.0 \mathrm{~h}$; amplitude: 12,934 \pm 7,538 photons/min; Fig. 2B, Table 1), and E22 uterus tissues (period: $24.2 \pm 0.7 \mathrm{~h}$; amplitude: 12,332 $\pm 9,063$ photons/min; Fig. 2C, Table 1). In both LD and $\mathrm{DD}$, the peak phases of the uterus were delayed by approximately 3-hours compared to those of the maternal SCN. No significant phase differences between the uterus and the maternal SCN were observed among non-pregnant and pregnant Perl-luc rats (one-way ANOVA, $p>0.05$, Table 1).

In both $\mathrm{LD}$ and $\mathrm{DD}$, neither the period nor amplitude of rhythmic uterus cultures depended on the stage of pregnancy (one-way ANOVA, $p>0.05$, Table 1). These data suggest that the biological clock in the uterus keeps a relatively stable circadian rhythmicity throughout pregnancy despite profound tissue remodeling in the uterus induced by sex steroids such as estrogen and progesterone to maintain pregnancy (Maeda et al. 2000).

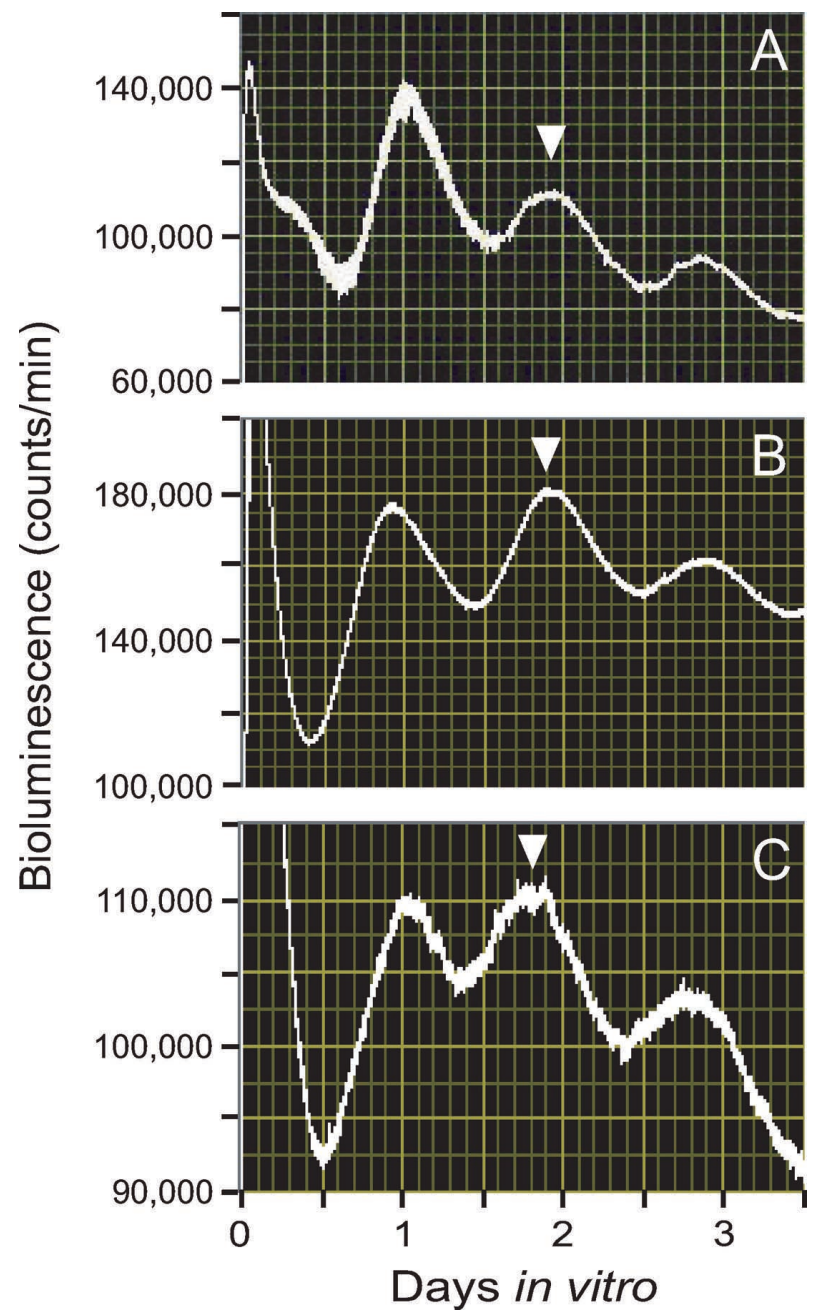

Fig. 1. Rhythms of Perl-luc light emission by uterus explants from Perl-luc rats in a light-dark cycle (LD). Shown are raw data from uterus tissues from ad lib-fed Perl-luc female rats in non-pregnancy (A), embryonic day 12 (B), and embryonic day 22 (C) in a 12:12 h LD cycle. The phase of the tissue in vivo has been considered to be best reflected by the phase of the peak in vitro during the first full subjective day ( 1 to 2.5 days after explant) as previously described (Yamazaki et al., 2000; Ohta et al., 2005). The phase of these peaks is consistent from animal to animal. Here, the phase statistically chosen is indicated by the inverted triangles.

Circadian rhythms of Perl expression in the rat placenta during pregnancy

Among structures of the placenta, we assayed portions of the labyrinthine throphoblast and the decidua from the E22 pregnant rats in LD. Although all the portions of placenta tissue expressed high levels of Perl-luc expressions, the labyrinthine throphoblast failed to show circadian rhythmicity. In contrast, the layer of decidual cells showed damped circadian oscillations for one to two cycles in vitro, followed by a prolonged falling phase (Fig. 3A, B).

The gradual reduction in amplitude of circadian oscillations in culture could result from a factor associated with cell culture such as a progressive cell death, desynchroniza- 
Table 1. Peaks, periods and amplitudes of Perl-luc uterus and SCN rhythms in nonpregnant and pregnant female rats in LD and DD.

\begin{tabular}{|c|c|c|c|c|c|}
\hline & & & Peak (hrs in vitro) & Period (h) & Amplitude (photons/min) \\
\hline \multirow[t]{6}{*}{ LD } & Uterus & Nonpregnant & $47.1 \pm 1.1$ & $24.1 \pm 0.8$ & $16,663 \pm 4,262$ \\
\hline & & E12 & $46.8 \pm 1.1$ & $24.1 \pm 0.5$ & $29,686 \pm 14,961$ \\
\hline & & E22 & $46.3 \pm 2.2$ & $25.1 \pm 0.7$ & $19,722 \pm 10,786$ \\
\hline & $\mathrm{SCN}$ & Nonpregnant & $43.4 \pm 0.8$ & $24.1 \pm 0.9$ & $1,722 \pm 410$ \\
\hline & & E12 & $42.8 \pm 1.1$ & $24.4 \pm 0.8$ & $1,446 \pm 962$ \\
\hline & & E22 & $43.8 \pm 1.3$ & $23.9 \pm 0.8$ & $1,412 \pm 761$ \\
\hline \multirow[t]{6}{*}{ DD } & Uterus & Nonpregnant & $48.0 \pm 1.8$ & $24.7 \pm 1.1$ & $19,013 \pm 11,303$ \\
\hline & & E12 & $46.6 \pm 0.7$ & $24.0 \pm 1.0$ & $12,934 \pm 7,538$ \\
\hline & & E22 & $46.1 \pm 1.5$ & $24.2 \pm 0.7$ & $12,332 \pm 9,063$ \\
\hline & $\mathrm{SCN}$ & Nonpregnant & $44.2 \pm 0.9$ & $24.1 \pm 0.7$ & $1,704 \pm 773$ \\
\hline & & E12 & $44.4 \pm 1.2$ & $24.0 \pm 0.7$ & $1,692 \pm 1,096$ \\
\hline & & E22 & $44.1 \pm 1.0$ & $23.7 \pm 0.5$ & $1,333 \pm 1,013$ \\
\hline
\end{tabular}

In vitro peaks, periods and amplitudes of Perl-luc uterus and SCN rhythms in non-pregnant $(n=5)$ and pregnant Perl-luc rats ( $n=5$ for both embryonic day 12 (E12) and embryonic day 12 (E22)) in a light-dark cycle (LD) and constant darkness (DD) ( $n=5$ for each, mean \pm S.D.). Peaks in vitro are defined as the peak times during the first full subjective day ( 1 to 2.5 days after explant) as previously described (Yamazaki et al. 2000; Ohta et al. 2005). No significant differences by one-way ANOVA $(p>0.05)$ between LD and DD in peaks and periods in both uterus and SCN rhythms.

tion of multiple sustained circadian oscillators, or coincident damping of individual oscillators. Among them, the possibility of cell death was rejected since forskolin, a Perl promoter stimulator through the CREB site, again evoked a transient increase in Perl-luc activites in the cultured tissues including both the labryrinthine throphoblast and the decidua, which once lost clear Perl-luc rhythmicity (data not shown).

We further assessed Perl mRNA expression in the placenta of E12 and E22 in both LD and DD cycles by in situ hybridization. No obvious circadian oscillation was observed in the labyrinth throughout pregnancy in both LD and DD (one-way ANOVA, $p>0.05$, Fig. 4-7). In contrast, circadian patterns of Perl mRNA expression were detected in the decidua of E12 and E22 in LD (one-way ANOVA, $p$ $<0.05$, Fig. 4-7). On E12 in DD, however, no circadian pattern was detected even in the decidua (one-way ANOVA, $p>0.05$, Fig. 6). These results suggest that circadian oscillations are maintained at least in the decidua, the maternally-originated tissue of the placenta, throughout pregnancy in LD.

The findings in our present study are consistent with previous reports in that no circadian rhythmicity was detected in adult tissues of the testis and thymus, which have the same property of on-going cellular differentiation as the labyrinth, a fetus-orignated tissue (Alvarez et al. 2003). The equal or relatively higher level of Perl mRNA expression in the labyrinth compared to the decidua can also be explained by the chronic elevated level of progesterone during pregnancy, which has been reported to continuously up-regulate Perl mRNA expression in vivo (He et al. 2007).

\section{Circadian rhythms of Perl expression in the maternal SCN during pregnancy}

The circadian expressions of the Perl-luc expressions in the maternal SCN were recorded simultaneously in nonpregnant and pregnant rats in LD (Fig. 8) and DD (Fig. 9). Perl-luc SCN rhythmicity remained unchanged between non-pregnant and pregnant rats (Table 1; one-way ANOVA, $p>0.05)$ in both LD and DD. These data suggest that the $\mathrm{SCN}$ of pregnant rats are not affected by various changes in hormonal environments induced by pregnancy and sustain a stable circadian rhythmicity throughout pregnancy.

\section{Discussion}

Two significant implications arise from these results. First, the circadian property of molecular clock in the uterus is stably maintained in almost the same manner from nonpregnancy, right through to the end stage of pregnancy, in regular light-dark (LD) cycles and constant darkness (DD). During pregnancy, the myometrium in the uterus undergoes profound remodeling including proliferation and decidulization in response to sex steroid hormones and implantation (Maeda et al. 2000). The uterus contains the receptors for both progesterone and estrogen and a previous report demonstrated that the Perl expression actually increases in the rat uterus after treatment with progesterone (P4) and a homologue of estrogen (estradiol: E2) in vitro (He et al. 2007). However, despite long-lasting increases in progesterone during gestation and a timely increase in estrogen before delivery (Maeda et al. 2000), the uterus kept a stable Perl-luc circadian rhythm throughout the pregnancy. The same story is also true with the maternal SCN. Although Per2 circadian rhythms in the SCN are reported to be 


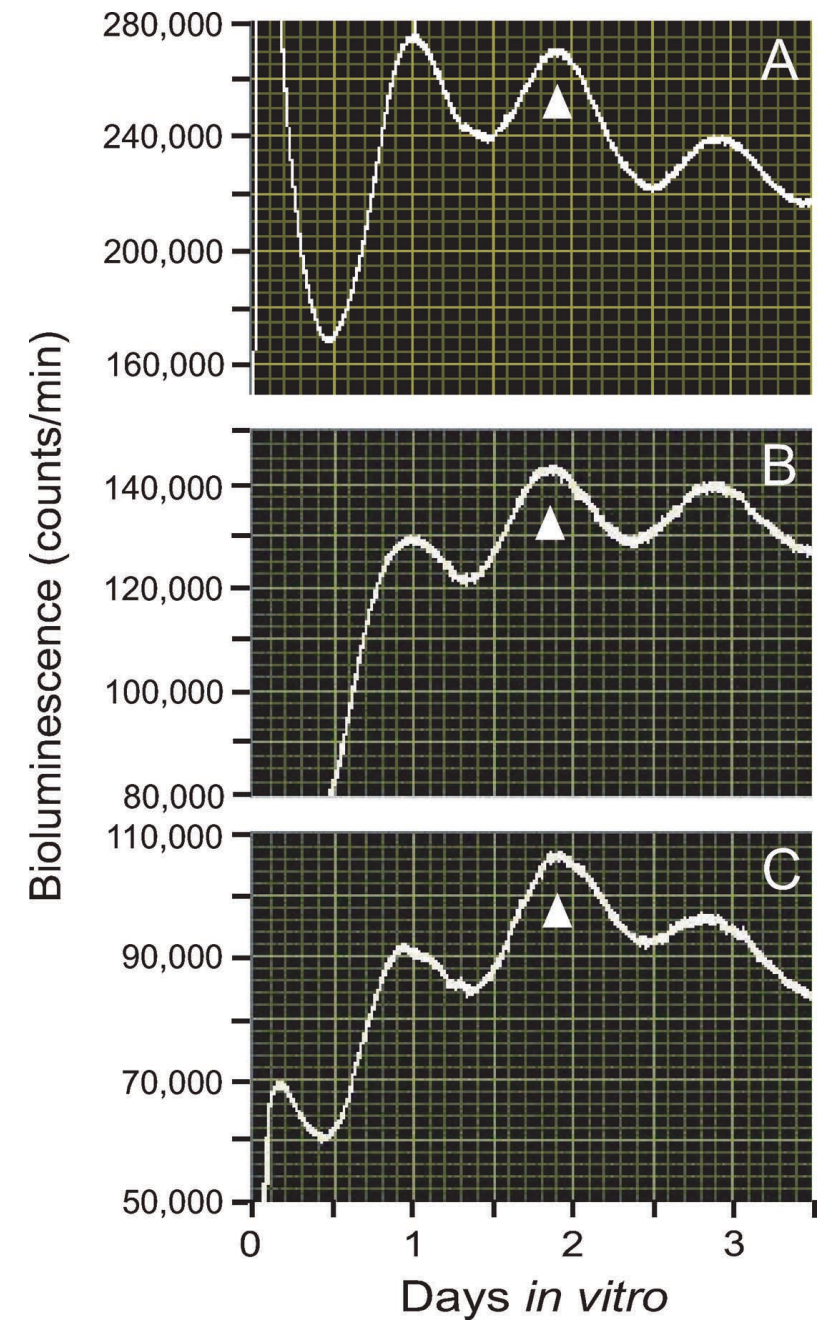

Fig. 2. Rhythms of Perl-luc light emission by uterus explants from Perl-luc rats in constant darkness (DD). The animals were exposed to DD for 2 consecutive days prior to explants to eliminate the lighting effects on the biological clock. Shown are raw data from uterus tissues from $a d$ lib-fed Per1-luc female rats in non-pregnancy (A), embryonic day 12 (B), and embryonic day 22 (C) in DD. Here, the phase statistically chosen is indicated by the triangles. For details, see legend of Fig. 1.

affected by an E2 implant in constant dark conditions (Nakamura et al. 2005), the property of Perl-luc circadian rhythmicity in the SCN was not affected by pregnancy in the present study, indicating that increases in circulating progesterone and estrogen are not likely to affect the molecular clock in either the uterus or maternal SCN during pregnancy. Probably, in a regular light-dark cycle, the effects of increasing sex hormones induced by pregnancy were countered by some complementary light-dependent resetting systems in the SCN. In addition, in non-pregnant rats in both LD and DD, circadian oscillation in the uterus peaked about 3 hours after maternal $\mathrm{SCN}$, indicating that the peripheral tissues such as the uterus have stable but different phases from the maternal SCN. The phase relationships between the uterus and maternal SCN were also the same as in

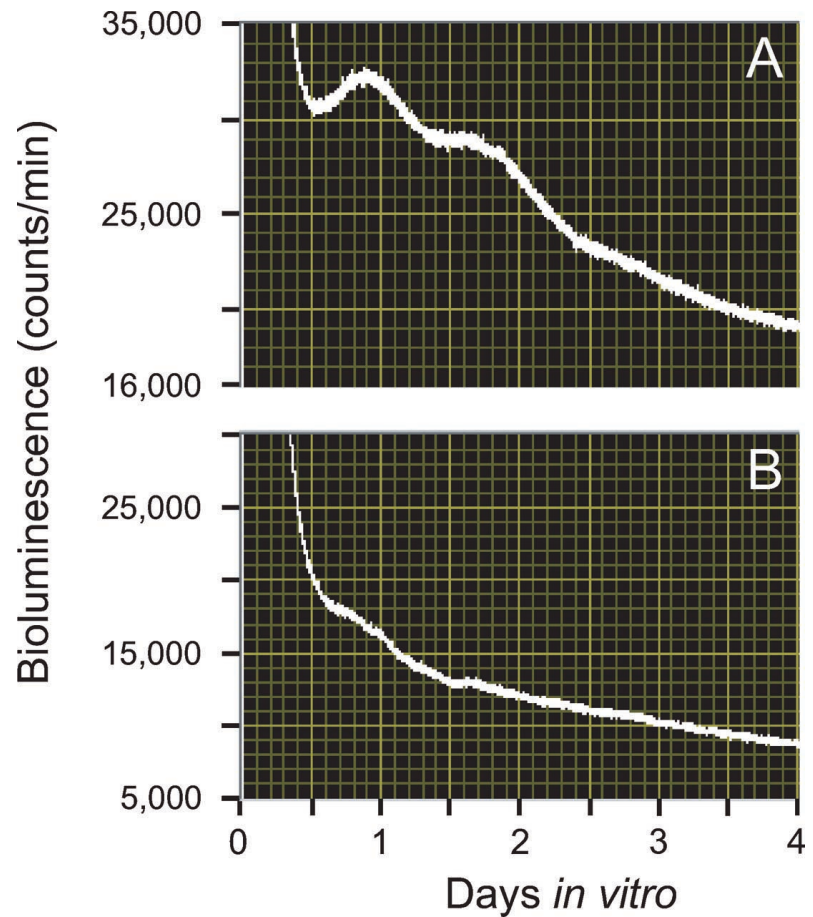

Fig. 3. Rhythms of Perl-luc light emission by placenta explants from Perl-luc rats in a light-dark cycle (LD). Shown are raw data from placenta tissues from $a d l i b$-fed Per1-luc female rats on embryonic day 22 in a $12: 12 \mathrm{~h}$ LD cycle. The decidua portion (A) shows damped circadian oscillations for one to two cycles in vitro, followed by a prolonged phase of falling bioluminescence while the labyrinth portion (B) does not show any clear oscillations. For details, see legend of Fig. 1.

pregnant rats (Table 1).

Second, in contrast to stable circadian rhythmicity in the uterus, the placenta does not have persistent Perl-luc rhythms. The placenta consists of both fetal- and maternaloriginated tissues. The present study examined the Perl-luc expressions of the labyrinth portion as fetal tissue and those of the decidua portion as maternal tissue. The labyrinth did not express Perl-luc circadian rhythms. The decidua did not have persistent oscillation but showed one to two cycles of Perl-luc expressions. This result is partly supported by an in vitro experiment, in which no continuous Per2-luc rhythms but only one peak in the uterine stromal cells were observed after decidualization induced by medroxyprogesterone acetate plus N6, 2-O-dibutyryl adenosine 3': 5'cyclic monoposphate (He et al. 2005). We further checked the Per 1 mRNA expression in the placenta by in situ hybridization. Again no obvious circadian oscillation was observed in the labyrinth but a circadian pattern of Perl mRNA expression was detected in the decidua. These results indicate that, unlike the uterus, the labyrinth, a fetusoriginated tissue of the placenta, does not have circadian properties in its physiological functions, while the decidua, a maternally originated tissue, has features of maintaining circadian oscillations. However, clear circadian expressions of $\mathrm{Mel} \mathrm{la} \mathrm{receptor} \mathrm{were} \mathrm{reported} \mathrm{in} \mathrm{the} \mathrm{placenta} \mathrm{(Lee} \mathrm{et} \mathrm{al.}$ 
A
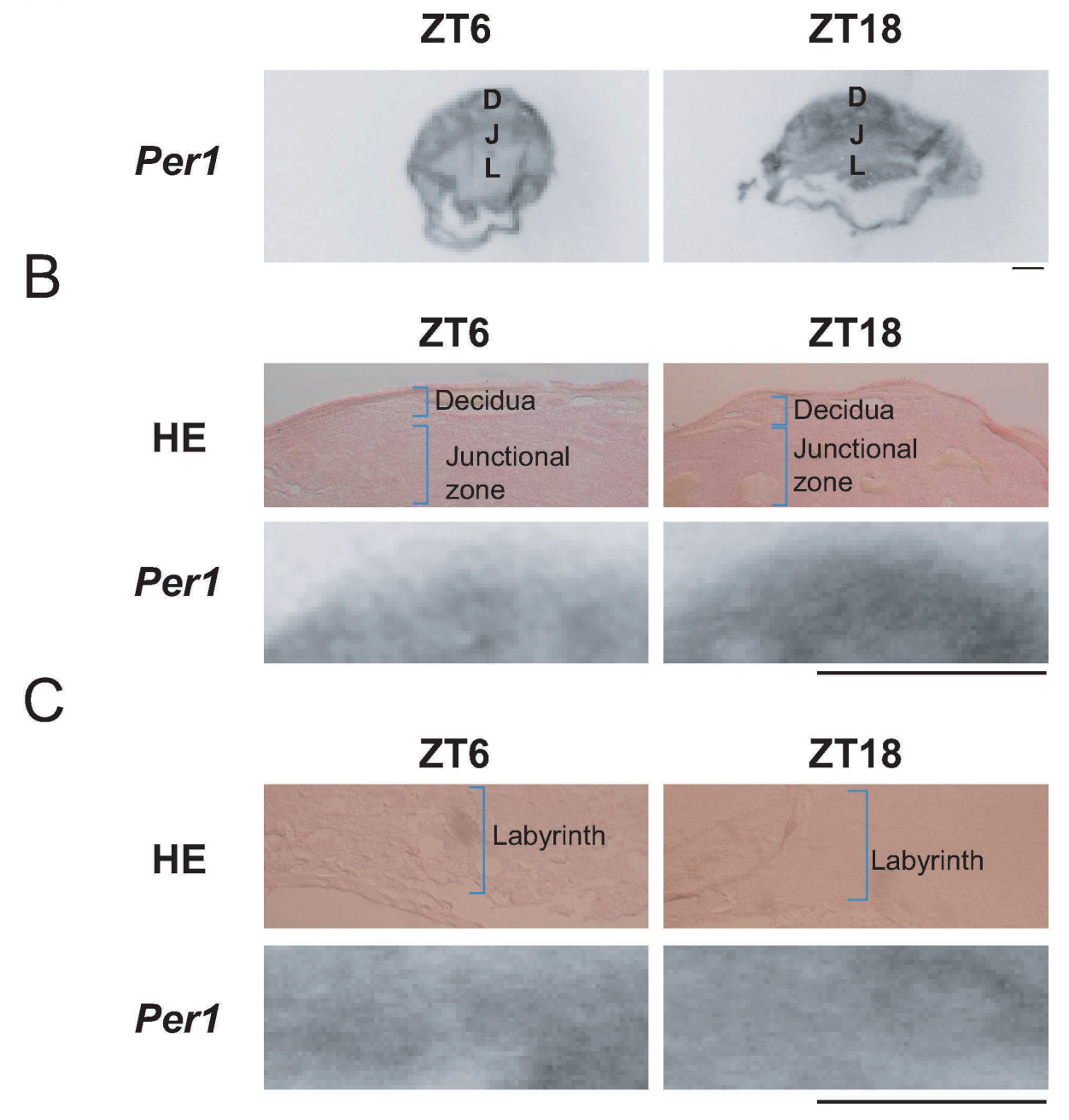

D

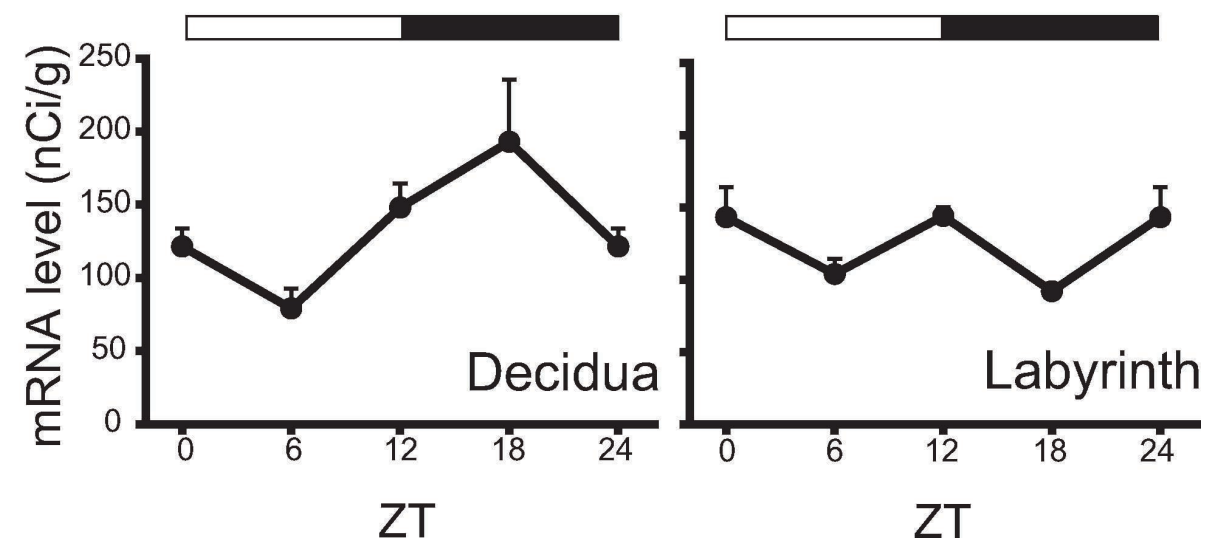

Fig. 4. The mRNA expression profiles of Perl gene in the placenta from wild-type rats in a ligh-dark cycle (LD) on E12. (A) Representative in situ hybridization autoradiograms showing the circadian change of Perl mRNA in the placenta at zeitgeber time (ZT) 6 and ZT18 on E12. Lights on and lights off are defined as ZT0 and ZT12, respectively. D, decidua; J, junctional zone; L, labyrinth. The bar $=1 \mathrm{~mm}$. (B) The decidua and the junctional zone in a placental section counterstained with hematoxylin and eosin (HE) after in situ hybridization. The bar $=1 \mathrm{~mm}$. (C) The labyrinth in a placental section counter-stained with HE after in situ hybridization. The bar $=1 \mathrm{~mm}$. (D) A daily profile of quantified Perl mRNA expression in the decidua and the labyrinth $(n=5$ at each sampling point, mean \pm S.E.). The horizontal white and black bars on the top of the graphs indicate the light and dark period, respectively. The decidua portion shows circadian rhythmicity in Perl mRNA (one-way ANOVA, $p<0.05$ ) while the labyrinth portion shows no circadian rhythmicity (one-way ANOVA, $p>0.05$ ). 
A

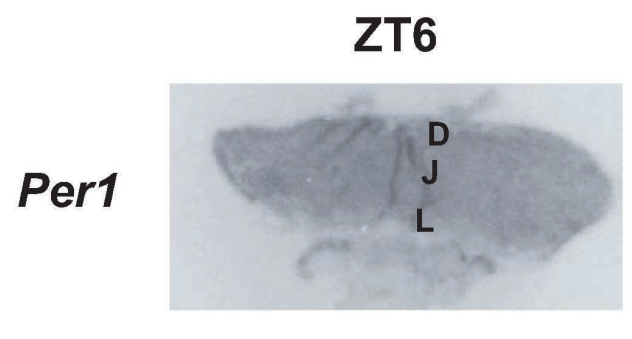

ZT6

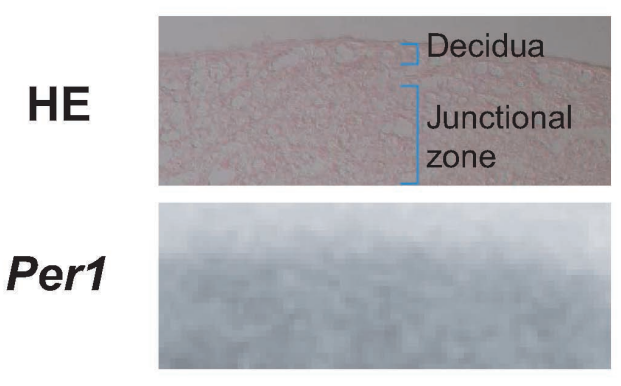

C

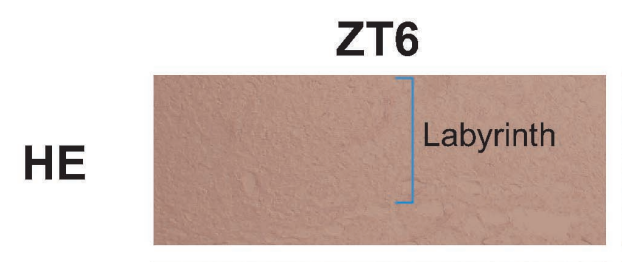

Per1

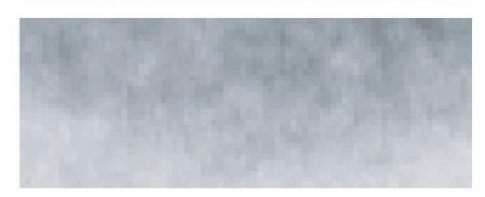

$\mathrm{D}$
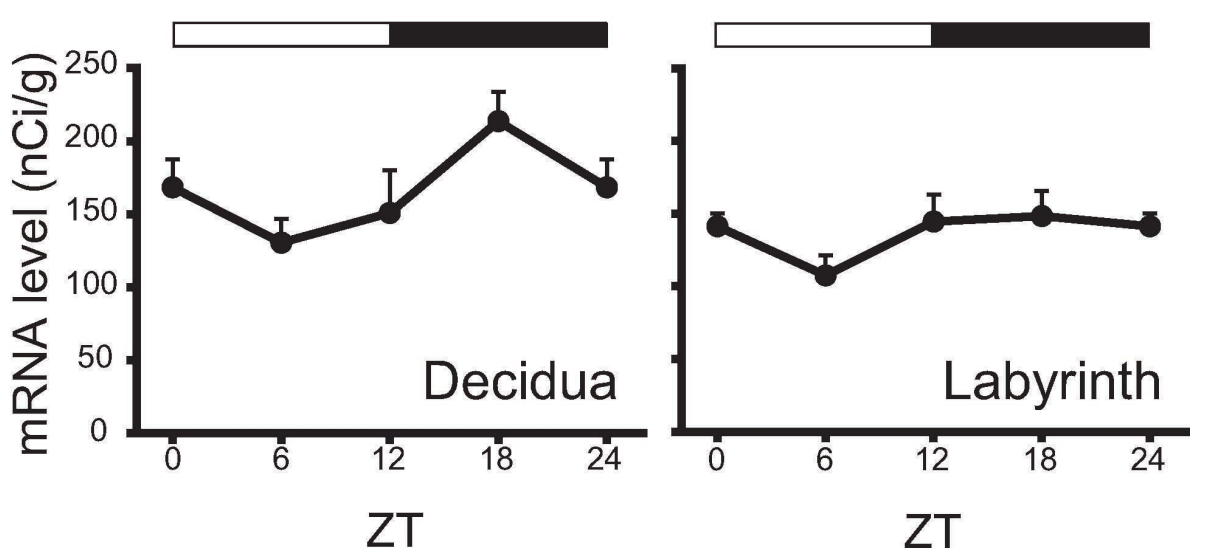

Fig. 5. The mRNA expression profiles of Perl gene in the placenta from wild-type rats in a light-dark cycle (LD) on E22. (A) Representative in situ hybridization autoradiograms showing the circadian change of Per1 mRNA in the placenta at zeitgeber time (ZT) 6 and ZT18 on E22. D, decidua; J, junctional zone; L, labyrinth. The bar $=1 \mathrm{~mm}$. (B) The decidua and the junctional zone in a placental section counter-stained with hematoxylin and eosin (HE) after in situ hybridization. The bar $=1 \mathrm{~mm}$. (C) The labyrinth in a placental section counter-stained with HE after in situ hybridization. The bar $=1$ mm. (D) A daily profile of quantified Perl mRNA expression in the decidua and the labyrinth $(n=5$ at each sampling point, mean \pm S.E.). The horizontal white and black bars on the top of the graphs indicate the light and dark period, respectively. The decidua portion shows circadian rhythmicity in Perl mRNA (one-way ANOVA, $p<0.05$ ) while the labyrinth portion shows no circadian rhythmicity (one-way ANOVA, $p>0.05$ ). 
A
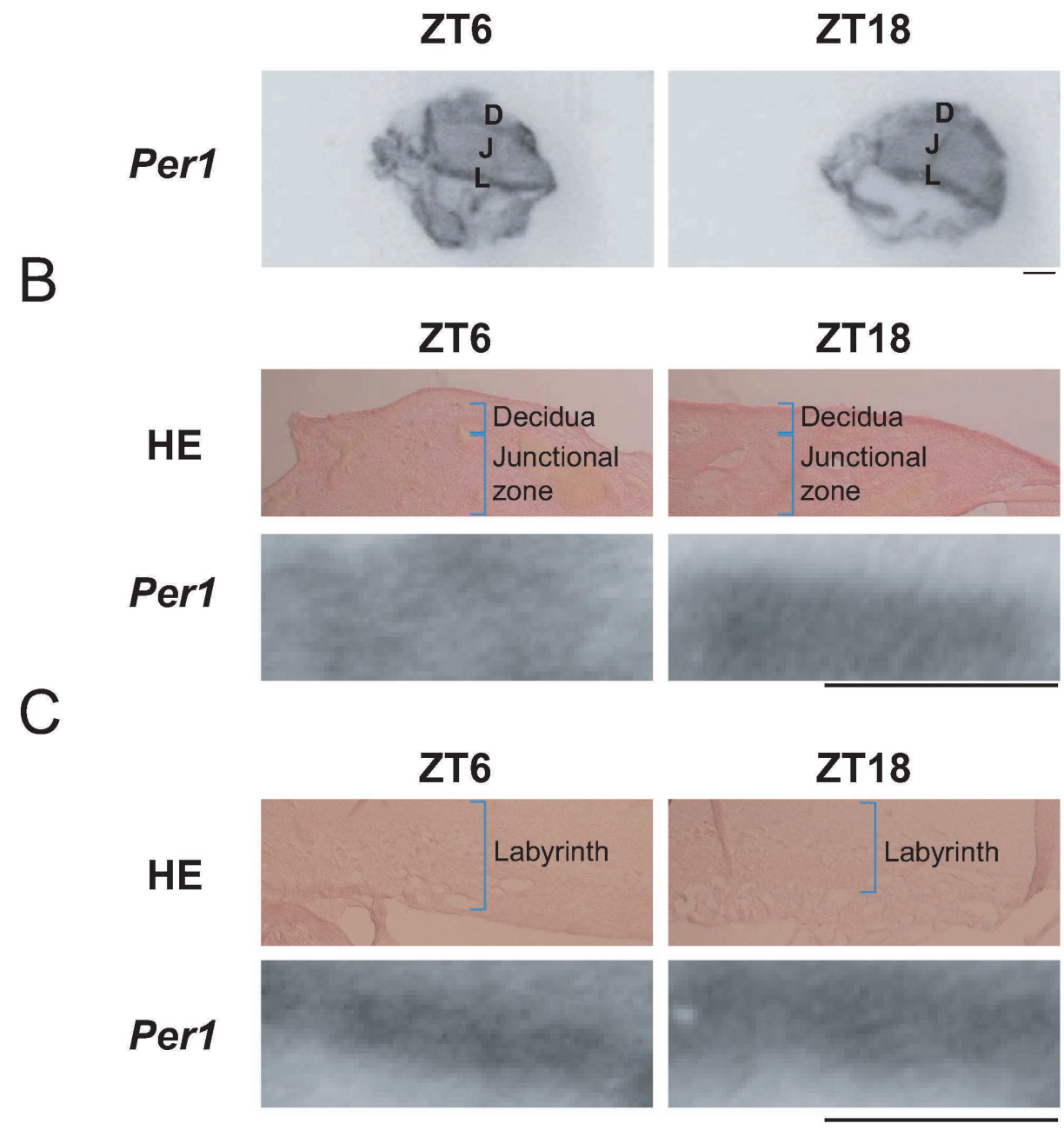

D
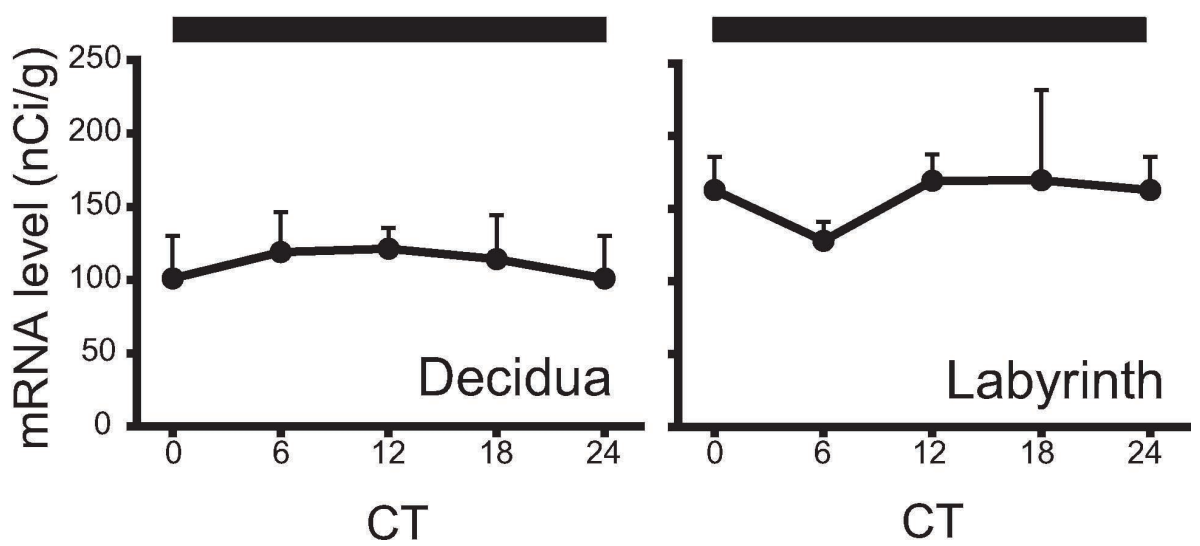

Fig. 6. The mRNA expression profiles of Perl gene in the placenta from wild-type rats in constant darkness (DD) on E12. (A) The animals were exposed to DD for 2 consecutive days prior to explants to eliminate the lighting effects on the biological clock. Representative in situ hybridization autoradiograms showing the circadian change of Perl mRNA in the placenta at CT 6 and CT18 on E12. CT 0 and 12 approximately correspond to dawn and dusk in the previous LD cycle, respectively. CT0 is defined as the termination of locomotor activity (the rats used are nocturnal animals). D, decidua; J, junctional zone; L, labyrinth. The bar $=1 \mathrm{~mm}$. (B) The decidua and the junctional zone in a placental section counterstained with hematoxylin and eosin (HE) after in situ hybridization. The bar $=1 \mathrm{~mm}$. (C) The labyrinth in a placental section counter-stained with HE after in situ hybridization. The bar $=1 \mathrm{~mm}$. (D) A daily profile of quantified Perl mRNA expression in the decidua and the labyrinth $(n=5$ at each sampling point, mean \pm S.E.). The horizontal black bar on the top indicates the 24-h continuous dark period. Both decidua and labyrinth portions show no circadian rhythmicity in Perl mRNA (one-way ANOVA, $p>0.05$ ). 
A
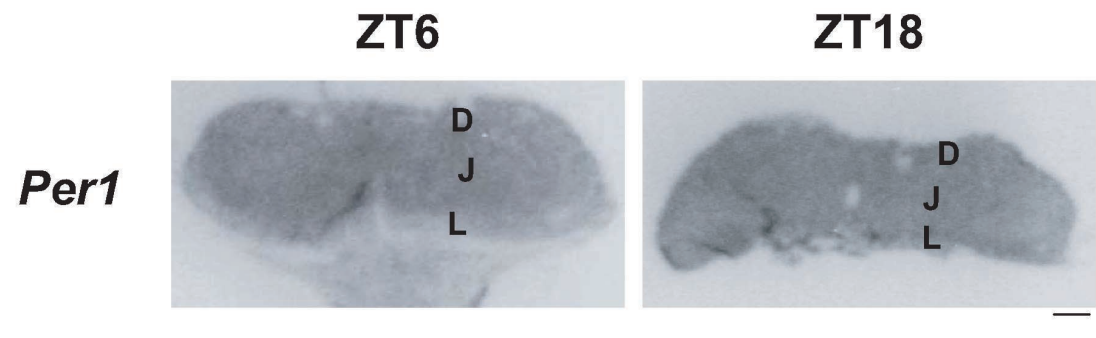

B
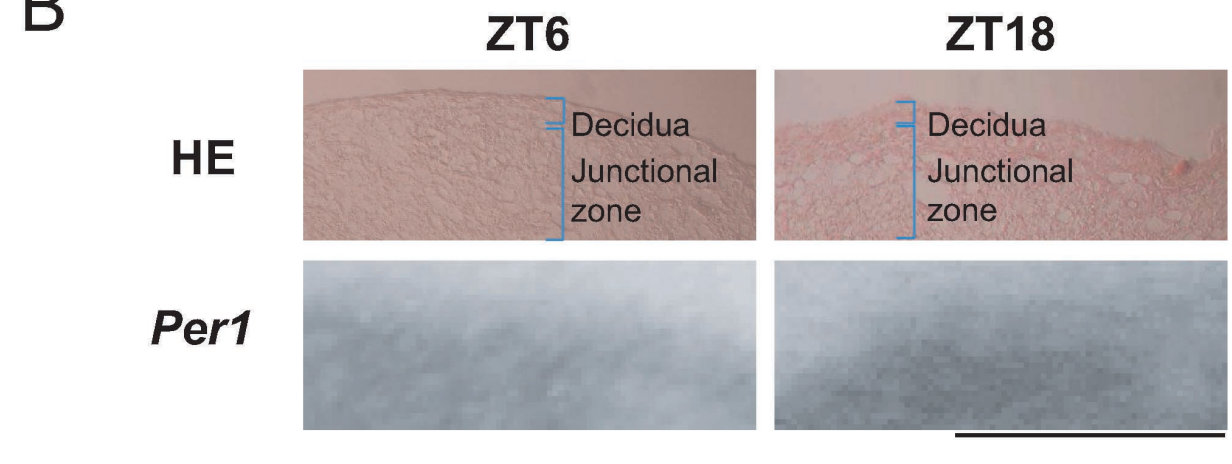

C
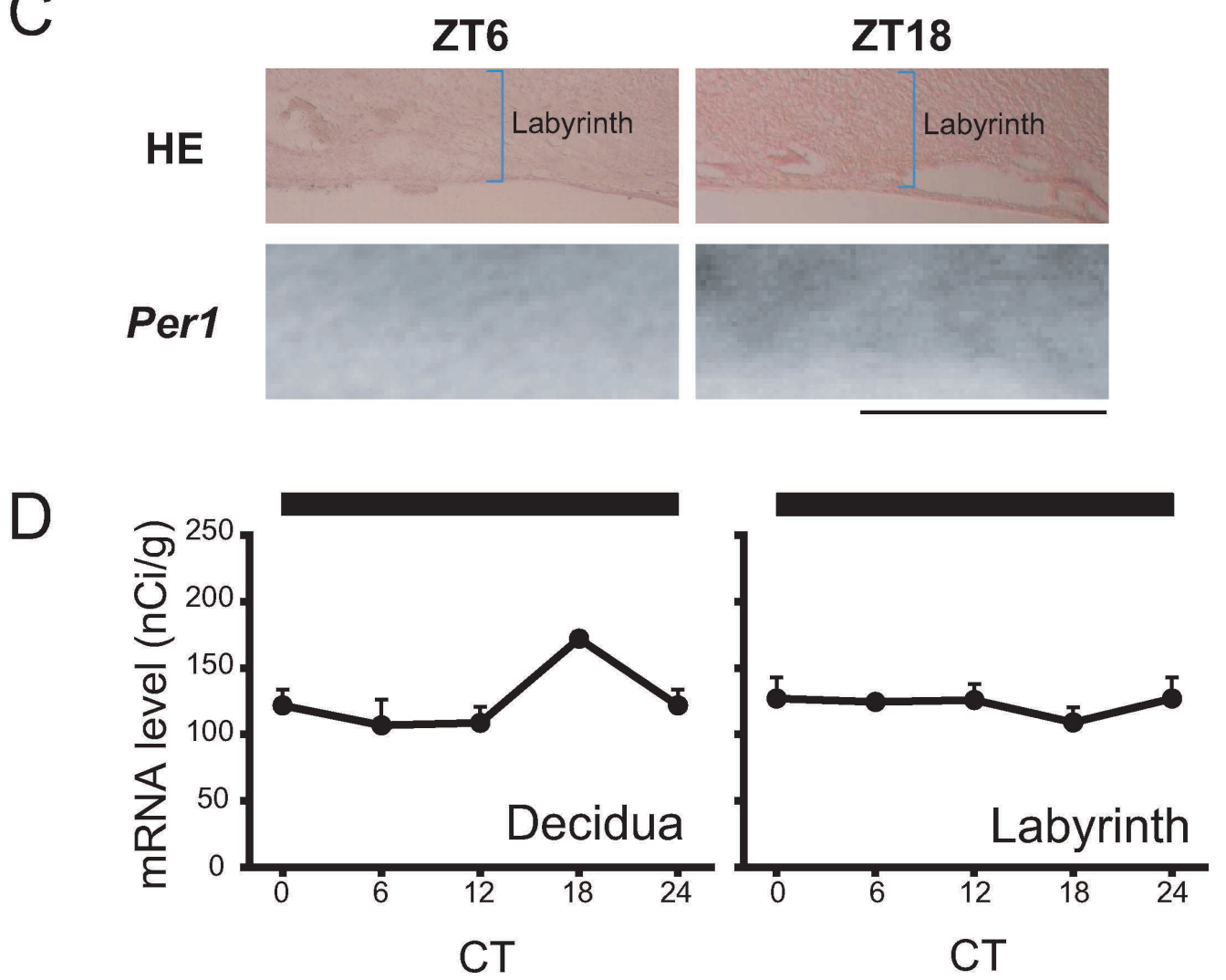

Fig. 7. The mRNA expression profiles of Per1 gene in the placenta from wild-type rats in constant darkness (DD) on E22. The animals were exposed to DD for 2 consecutive days prior to explants to eliminate the lighting effects on the biological clock. (A) The animals were exposed to DD for 2 consecutive days prior to explants to eliminate the lighting effects on the biological clock. Representative in situ hybridization autoradiograms showing the circadian change of Per1 mRNA in the placenta at CT 6 and CT18 on E22. D, decidua; J, junctional zone; L, labyrinth. The bar $=1 \mathrm{~mm}$. (B) The decidua and the junctional zone in a placental section counter-stained with hematoxylin and eosin (HE) after in situ hybridization. The bar $=1 \mathrm{~mm}$. (C) The labyrinth in a placental section counter-stained with HE after in situ hybridization. The bar $=1 \mathrm{~mm}$. (D) A daily profile of quantified Per 1 mRNA expression in the decidua and the labyrinth $(n=5$ at each sampling point, mean \pm S.E.). The horizontal black bar on the top indicates the 24-h continuous dark period. The decidua portion shows circadian rhythmicity in Perl mRNA (one-way ANOVA, $p<0.05$ ) while the labyrinth portion shows no rhythmicity (one-way ANOVA, $p>0.05$ ). 


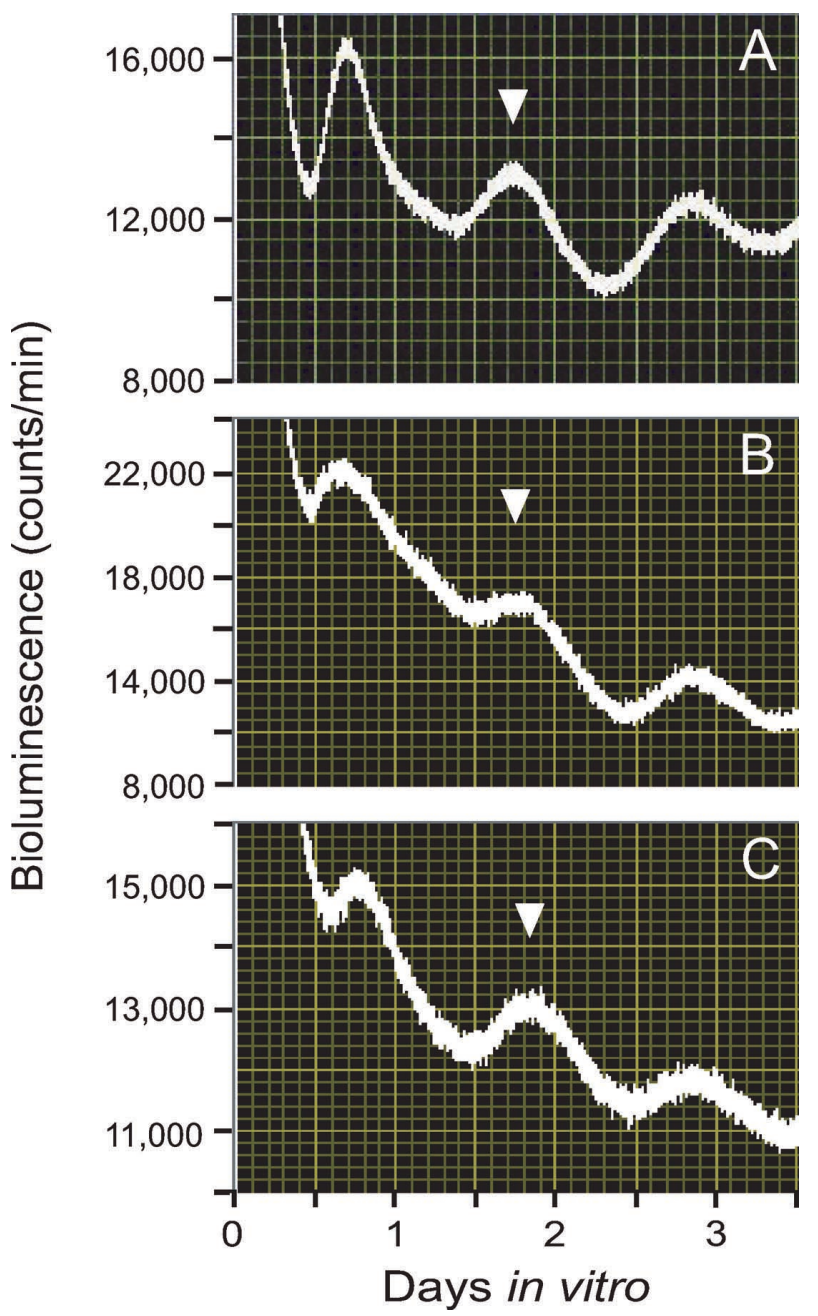

Fig. 8. Rhythms of Perl-luc light emission by maternal SCN explants from Per1-luc rats in a light-dark cycle. Shown are raw data from SCN tissues of Perl-luc ad lib-fed female rats in non-pregnancy (A), embryonic day 12 (B), and embryonic day $22(\mathrm{C})$ in a 12:12 h LD cycle. The phase statistically chosen is indicated by the inverted triangles.

2003). Since exogenous melatonin supply induces $\mathrm{Mel} \mathrm{la}$ receptor mRNA expression but does not affect clock gene mRNA expressions such as Period 2 in the fetal organs (Torres-Farfan et al. 2006), circadian secretion of maternal melatonin may directly cause circadian expression patterns of Mel la receptors but may not contribute to entrainment of circadian clock gene expressions in the labyrinth, a fetaloriginated tissue.

Previous studies have suggested that the fetus in the uterus uses maternal signals to adapt the fetal biological clock to the maternal environment (Reppert and Schwartz 1983; Davis and Gorski 1988; Parraguez et al. 1996; Kennaway 2002; Ohta et al. 2002, 2003, 2008; Sládek et al. 2004; Li and Davis 2005; Saxena et al. 2007). These signals are considered to be a variety of nonphotic stimuli including circadian fluctuations in hormonal and nutritional signals from the mother, placental filtering of those signals,

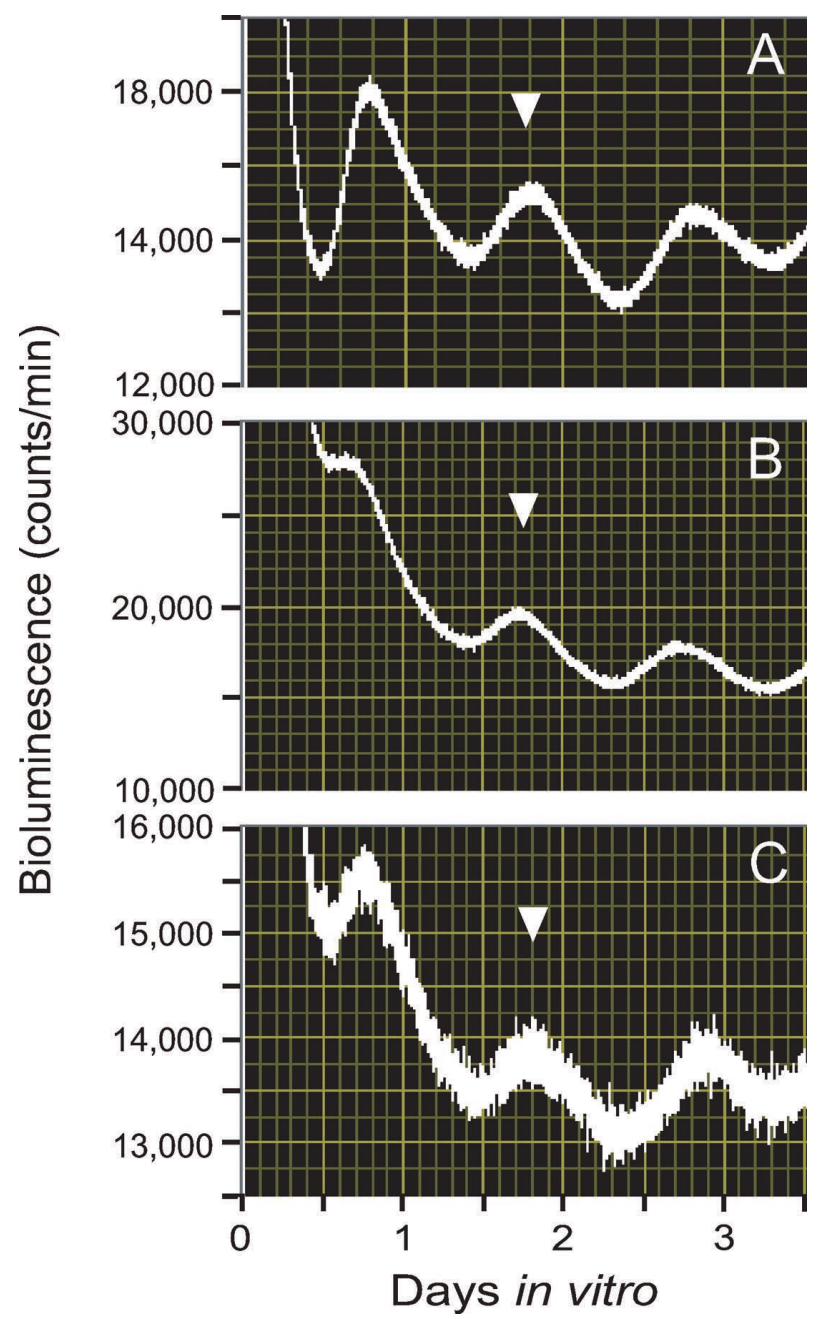

Fig. 9. Rhythms of Per1-luc light emission by maternal SCN explants from Perl-luc rats in constant darkness (DD). The animals were exposed to DD for 2 consecutive days prior to explants to eliminate the lighting effects on the biological clock. Shown are raw data from SCN tissues of ad lib-fed Per1-luc female rats in non-pregnancy (A), embryonic day 12 (B), and embryonic day 22 (C) in constant darkness (DD). The phase statistically chosen is indicated by the inverted triangles.

and uterine motility (Serón-Ferré et al. 2007). The present study further supports this idea particularly by first proving the existence of stable circadian clocks in the pregnant uterus and the decidua at the molecular level. Our study gives a basis to explore the molecular connections between circadian clocks in pregnant reproduction organs and their physiological functions and also suggests an importance of circadian environments for developing organisms to achieve their sound physiology (Mann et al. 1986; Miller et al. 1995; Mirmiran and Ariagno 2000; Brandon et al. 2002; Rivkees et al. 2004; Miller et al. 2004; Ohta et al. 2006, 2008).

\section{Acknowledgments}

We thank the Institute of Animal Experimentation at Tohoku University Graduate School of Medicine for use of its facilities 
and their technical assistance. We also thank Dr. Shin Yamazaki, Dr. Shanhai Xu, Mr. Seiji Suzuki, Mr. Yoshinori Takamatsu, Mr. Atsushi Kamoshida, Mr. Thor Masuda and Mr. David Egginton for their technical and editorial advice. This work was supported by Grants-in-Aid for Scientific Research (to H.O., K.O., and N.Y.) from the Ministry of Education, Science, Sports and Culture, the Ministry of Health, Labour and Welfare and New Energy and Industrial Technology Development Organization.; grants from Takeda Science Foundation, and Research Foundation for OptoScience and Technology (to H.O.).

\section{References}

Alvarez, J.D., Chen, D., Storer, E. \& Sehgal, A. (2003) Non-cyclic and developmental stage-specific expression of circadian clock proteins during murine spermatogenesis. Biol. Reprod., 69, 81-91.

Brandon, D.H., Holditch-Davis, D. \& Belyea, M. (2002) Preterm infants born at less than 31 weeks' gestation have improved growth in cycled light compared with continuous near darkness. J. Pediatr., 140, 192-199.

Davis, F.C. \& Gorski, R.A. (1988) Development of hamster circadian rhythms: role of the maternal suprachiasmatic nucleus. $J$. Comp. Physiol. [A], 162, 601-610.

Davis, F.C. \& Mannion, J. (1988) Entrainment of hamster pup circadian rhythms by prenatal melatonin injections to the mother. Am. J. Physiol., 255, R439-448.

Dolatshad, H., Campbell, E.A., O'hara, L., Maywood, E.S., Hastings, M.H. \& Johnson, M.H. (2006) Developmental and reproductive performance in circadian mutant mice. Hum. Reprod., 21, 68-79.

Hastings, M.H., Reddy, A.B. \& Maywood, E.S. (2003) A clockwork web: circadian timing in brain and periphery, in health and disease. Nat. Rev. Neurosci., 4, 649-661.

Hayasaka, N., Yaita, T., Kuwaki, T., Honma, S., Honma, K., Kudo, T. \& Shibata, S. (2007) Optimization of dosing schedule of daily inhalant dexamethasone to minimize phase shifting of clock gene expression rhythm in the lungs of the asthma mouse model. Endocrinology, 148, 3316-3326.

He, P.J., Hirata, M., Yamauchi, N., Hashimoto, S. \& Hattori, M.A. (2005) The disruption of circadian clockwork in differentiating cells from rat reproductive tissues as identified by in vitro real-time monitoring system. J. Endocrinol., 193, 413-420.

He, P.J., Hirata, M., Yamauchi, N. \& Hattori, M.A. (2007) Up-regulation of Perl expression by estradiol and progesterone in the rat uterus. J. Endocrinol., 194, 511-519.

Hida, A., Koike, N., Hirose, M., Hattori, M., Sakaki, Y. \& Tei, H. (2000) The human and mouse Period1 genes: five well-conserved E-boxes additively contribute to the enhancement of mPer1 transcription. Genomics, 65, 224-233.

Hirao, A., Tahara, Y., Kimura, I. \& Shibata, S. (2009) A balanced diet is necessary for proper entrainment signals of the mouse liver clock. PLoS One, 4, e6909.

Illnerová, H., Buresová, M. \& Presl, J. (1993) Melatonin rhythm in human milk. J. Clin. Endocrinol. Metab., 77, 838-841.

Kennaway, D.J. (2002) Programming of the fetal suprachiasmatic nucleus and subsequent adult rhythmicity. Trends Endocrinol. Metab., 13, 398-402.

Lee, C.K., Moon, D.H., Shin, C.S., Kim, H., Yoon, Y.D., Kang, H.S., Lee, B.J. \& Kang, S.G. (2003) Circadian expression of Mel1a and PL-II genes in placenta: effects of melatonin on the PL-II gene expression in the rat placenta. Mol. Cell. Endocrinol., 200, 57-66.

Li, X. \& Davis, F.C. (2005) Developmental expression of clock genes in the Syrian hamster. Brain Res. Dev. Brain Res., 158, $31-40$.

Maeda, K., Ohkura, S. \& Tsukamura, H. (2000) Physiology of reproduction. In The Laboratory Rat, 1st ed., edited by Krinke GJ. Academic Press, San Diego, PA, pp. 145-176.
Mann, N.P., Haddow, R., Stokes, L., Goodley, S. \& Rutter, N. (1986) Effect of night and day on preterm infants in a newborn nursery: randomised trial. Br. Med. J. (Clin. Res. Ed), 293, 12651267.

Maywood, E.S., O’Neill, J.S., Chesham, J.E. \& Hastings, M.H. (2007) Minireview: The circadian clockwork of the suprachiasmatic nuclei-analysis of a cellular oscillator that drives endocrine rhythms. Endocrinology, 148, 5624-5634.

Miller, B.H., Olson, S.L., Turek, F.W., Levine, J.E., Horton, T.H. \& Takahashi, J.S. (2004) Circadian clock mutation disrupts estrous cyclicity and maintenance of pregnancy. Curr. Biol., 14, 1367-1373.

Miller, C.L., White, R., Whitman, T.L., O'Callaghan, M.F. \& Maxwell, S.E. (1995) The effects of cycled versus noncycled lighting on growth and development in preterm infants. Infant Behav. Dev., 18, 87-95.

Mirmiran, M. \& Ariagno, R.L. (2000) Influence of light in the NICU on the development of circadian rhythms in preterm infants. Semin. Perinatol., 24, 247-257.

Moriya, T., Horikawa, K., Akiyama, M. \& Shibata, S. (2000) Correlative association between $\mathrm{N}$-methyl-D-aspartate receptormediated expression of period genes in the suprachiasmatic nucleus and phase shifts in behavior with photic entrainment of clock in hamsters. Mol. Pharmacol., 58, 1554-1562.

Nakamura, T.J., Moriya, T., Inoue, S., Shimazoe, T., Watanabe, S., Ebihara, S. \& Shinohara, K. (2005) Estrogen differentially regulates expression of Perl and Per2 genes between central and peripheral clocks and between reproductive and nonreproductive tissues in female rats. J. Neurosci. Res., 82, 622-630.

Ohta, H., Honma, S., Abe, H. \& Honma, K. (2002) Effects of nursing mothers on $r$ Perl and rPer 2 circadian expressions in the neonatal rat suprachiasmatic nuclei vary with developmental stage. Eur. J. Neurosci., 15, 1953-1960.

Ohta, H., Honma, S., Abe, H. \& Honma, K. (2003) Periodic absence of nursing mothers phase-shifts circadian rhythms of clock genes in the suprachiasmatic nucleus of rat pups. Eur. J. Neurosci., 17, 1628-1634.

Ohta, H., Mitchell, A.C. \& McMahon, D.G. (2006) Constant light disrupts the developing mouse biological clock. Pediatr. Res., 60, 304-308.

Ohta, H., Xu, S., Moriya, T., Iigo, M., Watanabe, T., Nakahata, N., Chisaka, H., Hanita, T., Matsuda, T., Ohura, T., Kimura, Y., Yaegashi, N., Tsuchiya, S., Tei, H. \& Okamura, K. (2008) Maternal feeding controls fetal biological clock. PLoS One, 3, e2601.

Ohta, H., Yamazaki, S. \& McMahon, D.G. (2005) Constant light desynchronizes mammalian clock neurons. Nat. Neurosci., 8 , 267-269.

Parraguez, V.H., Valenzuela, G.J., Vergara, M., Ducsay, C.A., Yellon, S.M. \& Serón-Ferré, M. (1996) Effect of constant light on fetal and maternal prolactin rhythms in sheep. Endocrinology, 137, 2355-2361.

Reppert, S.M. \& Schwartz, W.J. (1983) Maternal coordination of the fetal biological clock in utero. Science, 220, 969-971.

Rivkees, S.A., Mayes, L., Jacobs, H. \& Gross, I. (2004) Restactivity patterns of premature infants are regulated by cycled lighting. Pediatrics, 113, 833-839.

Saxena, M.T., Aton, S.J., Hildebolt, C., Prior, J.L., Abraham, U., Piwnica-Worms, D. \& Herzog, E.D. (2007) Bioluminescence imaging of periodl gene expression in utero. Mol. Imaging, 6 , 68-72.

Serón-Ferré, M., Valenzuela, G.J. \& Torres-Farfan, C. (2007) Circadian clocks during embryonic and fetal development. Birth Defects Res. C Embryo Today, 81, 204-214.

Shimomura, H., Moriya, T., Sudo, M., Wakamatsu, H., Akiyama, M., Miyake, Y. \& Shibata, S. (2002) Differential daily expression of Perl and Per2 mRNA in the suprachiasmatic nucleus of fetal and early postnatal mice. Eur. J. Neurosci., 13, 687-693. 
Sládek, M., Sumová, A., Kováciková, Z., Bendová, Z., Laurinová, K. \& Illnerová, H. (2004) Insight into molecular core clock mechanism of embryonic and early postnatal rat suprachiasmatic nucleus. Proc. Natl. Acad. Sci. USA, 101, 6231-6236.

Torres-Farfan, C., Rocco, V., Monsó, C., Valenzuela, F.J., Campino, C., Germain, A., Torrealba, F., Valenzuela, G.J. \& Serón-Ferré, M. (2006) Maternal melatonin effects on clock gene expression in a nonhuman primate fetus. Endocrinology, 147,
4618-4626

Weaver, D.R. \& Reppert, S.M. (1986) Periodic feeding of SCNlesioned pregnant rats entrains the fetal biological clock. Brain Res. Dev. Brain Res., 46, 291-296.

Yamazaki, S., Numano, R., Abe, M., Hida, A., Takahashi, R., Ueda, M., Block, G.D., Sakaki, Y., Menaker, M. \& Tei, H. (2000) Resetting central and peripheral circadian oscillators in transgenic rats. Science, 288, 682-685. 